\title{
33. STRUCTURE AND DYNAMICS OF THE GALACTIC SYSTEM (STRUCTURE ET DYNAMIQUE DU SYSTEME GALACTIQUE)
}

PRESIDENT: R. Wielen VICE-PRESIDENT: W.B. Burton ORGANIZING COMMITTEE: L. Blitz, W. Iwanowska, E.K. Kharadze, G.G. Kuzmin, D. Lynden-Be11, G. Lynga, M. Mayor, M. Miyamoto

\section{Introduction}

Several authors have contributed to this report: L. Blitz (Section V), W.B. Burton (Sections IIIB and IVB), J. Einasto (Section VII), B. Fuchs (Sections VIC and VID), W. Hermsen (Section VIF), G. Lynga (Sections IIIA and IVA), M. Mayor (Section II), M. Miyamoto (Sections VIB and VIE) and R. Wielen (Sections I, VIA, and editing). The layout of this report follows previous practice. The galactic center is included in Sections IV and V. The references are, as far as possible, coded by their numbers (VV.CCC.NNN) in the bibliography "Astronomy and Astrophysics Abstracts" (AAA). VV identifies the volume of AAA, while CCC.NNN gives the subject category and the serial number within that volume.

The field of galactic research has been very active in the last three years. During the triennium under review, many conferences of importance for galactic research have taken place. Among the IAU Symposia or Colloquia, we mention IAU Symposium No. 100 "Internal Kinematics and Dynamics of Galaxies" (33.012.004), IAU Symposium No. 105 "Observational Tests of the Stellar Evolution Theory" (37.012.078), IAU Symposium No. 106 "The Milky Way as a Galaxy", IAU Symposium No. 113 "Dynamics of Star Clusters", IAU Colloquium No. 76 "Nearby Stars and the Luminosity Function" (34.012.065), and IAU Colloquium No. 78 "Astronomy with Schmidt-type Telescopes" $(37.012 .077)$.

Among other relevant meetings were "Giant Molecular Clouds in the Galaxy" (29.012.012), "Galactic Evolution" (29.012.024), "Galaktische Struktur und Entwicklung" (29.012.039), "Structure and Evolution of Normal Galaxies" (30.012.010), "The Phases of the Interstellar Medium" (30.012.018), "Scientific Aspects of the HIPPARCOS Space Astrometry Mission" (31.012.041), "Morphology and Dynamics of Galaxies" (32.012.032), "The Galactic Center" (32.012.076), "Kinematics, Dynamics and Structure of the Milky Way" (33.012.016), "Evolution of Old Stellar Populations in Galaxies" (33.012.028), "Theoretical Aspects on Structure, Activity, and Evolution of Galaxies" I (33.012.048) and II (37.012.034), "Galactic Astrophysics and Gamma-Ray Astronomy" (34.012.016), "Surveys of the Southern Galaxy" (34.012.023), "Star Clusters and Associations and their Relation to the Evolution of the Galaxy" (34.012.068), "Formation and Evolution of Galaxies and Large Structures in the Universe" (37.012.002), "Statistical Methods in Astronomy" (37.012.003), and "Population Synthesis" (37.012.081).

A text book on the Galaxy, "Bau und Physik der Galaxis", has been written by Scheffler and E1sässer (32.003.108). Subvolume $2 \mathrm{c}$ of the Landolt-Börnstein (32.003. 055) contains articles on our Galaxy by Gliese, Scheffler and Wielen. Among relevant general review papers, in addition to those published in the proceedings of the conferences 1 isted above, are those of Bok (34.155.056), Pagel and Edmunds (30.155.039), Mould (32.155.027), York (32.155.028), Binney (32.151.041), Bignami and Hermsen (34.143.025), Kron (32.151.066), Güsten and Mezger (34.155.081), Oort (32.155.025), Delhaye $(32.155 .050)$, Downes and Güsten $(32.155 .053)$, Buser (32.155. 054), Neckel and Klare (32.155.055), and Contopoulos (34.151.103). 


\section{Basic Data and Calibrations}

\section{A. BASIC DATA}

It would hardly be possible to present in this summary an exhaustive 1 ist of all the important data for studies of galactic structure that were collected during the corresponding triennium. However, we tried to keep this report as complete as possible.

The Centre de Données Stellaires (CDS) has continued to publish basic information for galactic research. Their information bulletin summarizes the available information as well as the catalogues (Bu1l. Inf. Cent. Données Stellaires 21 to 25) $(30.002 .030,30.002 .095,31.002 .037,31.002 .033,32.002 .114,34.002 .021,34$. $002.026,34.002 .124,34.002 .024)$. Many catalogues are available on magnetic tape or microfiche. Direct telephone connection is now available between observatories and the CDS data base. In the US, astronomical data can be obtained from the Astronomical Data Centre; information on available data are given in 30.002.021, 30.002. $023,30.002 .034,30.002 .043,31.002 .032,31.002 .051,31.002 .081$ to $31.002 .095,32$. 002.082 to $32.002 .088,34.002 .020,34.002 .087$. See a1so "Automated data retrieval in astronomy", IAU Colloquium No. $62(32.012 .002)$.

A large set of basic data for galactic structure is expected from the future HIPPARCOS/TYCHO space mission: star positions, parallaxes, proper motions, magnitudes ("The scientific aspects of the HIPPARCOS Space Astrometry mission", 34.012. 070). During the last triennium, intensive preparative work has been done in relation with the input catalogue (see INCA News letters, contact C. Turon, Obs, de Meudon).

Schmidt-Kaler in Landolt-Börnstein (31.003.064) presents a survey, a critical appraisal of relevant work (up to the end of 1981) and tabulations of "Physical Parameters of the Stars".

Among new photometric catalogues or investigations we can mention: "The third catalogue of stars measured in the Geneva Observatory photometric system", giving 7-colour photometry for 14633 stars, presented by Rufener (30.002.008); "An infrared astronomical data base and catalogue of infrared observations" ( $1 \mu \mathrm{m}-1000 \mu \mathrm{m})$ by Schmitz, Gezari and Mead (30.002.037); McClure and Forrester have prepared a catalogue of homogeneous photometry of 2196 bright $G$ and $K$ stars in the DDO systems (30.002.077), and Meylan has established a compilation of measurements in the DDO photometric system, available on magnetic tape for 2911 stars (31.002.003); Upgren, Dawson and Lu have obtained DDO photometry for a number of dK-M stars $(30.113 .051)$.

25654 photoelectric measurements in the UBV photometric system published from 1976 to 1982 have been collected by Mermilliod (34.002.121). All photometric data were collected by Hauck in a general catalogue of photometric data (31.002.022); red and infrared photoelectric magnitudes in the Kron photometric system have been compiled by Jasniewicz in a. homogeneous catalogue on magnetic tape (31.002.066). Positions and uvby $\beta$ photometry are presented for about 1000 0-F8 stars towards the north galactic pole by Hill, Barnes and Hilditch (33.002.066, 33.002.067). UBVr magnitudes of more than 13000 stars at high galactic latitude have been prepared on magnetic tape by Weistrop (34.002.017). 01son has measured uvby $\beta$ photometry of 14816 A5 to Go stars brighter than 8.3 (34.002.029). Reed and Fitzgerald have co1lected UBV photometry of 610 stars in Puppis (34.002.028). The colours, magnitudes and parallaxes of 1077 nearby stars belonging to the 1969 edition of Gliese's catalogue have been collected by Grenon and Rufener with an up-to-date spectral classification $(30.113 .017)$. 
RGU photometry has been measured and analyzed: of fields in the direction of the Scutum cloud, Karaali (31.113.073); in an anticentre field around NGC 2129, Topaktas (31.113.074); in fields near the galactic centre, Wiedemann (32.113.018), Fenkart et al. (33.113.019), Becker, Fang (31.155.048); in a field of the galactic bulge, Spaenhauer et a1. (33.113.027); in a field in Cassiopeia, Fenkart, Kandemir (34.113.035); in a field in the large Sagittarius Cloud, Topaktas (30.155.032), and in a field in Centaurus, Spaenhauer, Fang (31.155.008). As tests of galactic models, the importance of these colour and magnitude counts is certainly increasing (see also 33.002 .044$)$. From RGU photometry of 1810 stars down to the 1 imiting magnitude $\mathrm{G}=19$, density gradients for disc and halo stars have been derived by Becker and Karali, as well as the luminosity function of m.s stars of population I within 400 to $800 \mathrm{pc}(33.155 .068)$.

Vilnius photometric determinations have been made for halo stars and stars at high galactic latitudes by Speranskas, Bartkevicius, Zdanavicius and Bartasinté $(31.113 .079,31.113 .080,31.113 .081)$. uvby photometry was used in many investigations, for example: by Degewij in McCormick proper motion fields (31.113.089), by Westin for 355 O-AO type stars near the galactic equator (32.113.003), by Hil1 and Barnes for about $700 \mathrm{AF}$ stars in the region of the north galactic pole (33.113.070, 33.113.071), by McFadzean, Hilditch and Hill for 572 O-F stars near the south galactic pole (34.113.023). UBV and $H B$ photometry of luminous stars between $1=335^{\circ}$ and $6^{\circ}$ was carried out by Dachs, Kaiser, Mikolov and Sherwood for 316 oB stars ( 32.113. 031). UBV photometry has been determined by Pier for $234 \mathrm{AB}$ stars in the southern halo (32.113.046), by Oja for 320 FK4 and FK4 supp1. stars (33.113.028).

A set of 223 stars was observed to provide new UBVRI standard stars (33.113. 003); V-R observations and effective temperatures are given for 90 extremely metaldeficient red giants by Stone (33.113.011). New UBVRI photometry is presented by Fernie for more than 900 supergiants $(33.113 .042)$. Near-infrared photometry (JHKLM) of 203 bright southern stars from various sources has been transformed by Koornneef into one homogeneous data set (33.113.025).

Schuster and Alvarez have observed Be and shell stars with the 13-colour photometric system (33.113.012), and Mendoza et al. have carried out a narrowband study for 228 04-B8-type stars (33.113.013).

A list of almost 2000 near-infrared sources in the galactic plane brighter than 6.5 in the $\mathrm{K}$-band has been published by Kawara et a1. (34.133.019). An ana1ysis of the stellar component of the Galaxy as seen by the AFGL Infrared Sky Survey has been reported by Grasdalen et al. and Kleinmann, Gillet and Joyce (34.155.065, $30.133 .008)$.

Depenchuk and Komarov, discussing the homogeneity of spectrophotometric standards, have stressed the necessity of a new absolute calibration of $\alpha$ Lyr in the UV and b1ue spectral regions (31.113.023). Secondary standard stars for absolute spectrophotometry based on an adopted absolute spectral energy distribution for the primary standard $\alpha$ Lyr are proposed by Oke and Gunn (33.113.017). A determination of the absolute monochromatic flux from Vega at $\lambda=2.20$ and $3.80 \mu \mathrm{m}$ through comparison with a furnace has been carried out (33.113.031, 34.113.039). Gunn and Stryker have presented a spectrophotometric catalogue of 175 stars covering a complete range of spectral types and luminosity classes for the wavelength region $3130<\lambda<10800 \&(33.002 .042)$.

Preliminary results on the calibration of the RGU photometric system have been presented by Thévenin, Spaenhauer and Foy (34.113.006); the relationship between the Johnson and Kron-Cousins VRI photometric systems can be found in (34.113.055). 
Basic data for all calibrations are certainly also the colours and absolute magnitude of the Sun. A new direct measurement gives $B-V=0.636, U-B=0.183$, and $M_{V}=-26.74$. uvby measurements show that the Sun seems to be somewhat metal-poor (Tüg and Schmidt-Kaler, 31.031.515).

Newly determined radial velocities, magnitudes and colours are given for 339 stars in the southern hemisphere (31.113.072), and spectral classification and photometry have been established for 221 barium stars (33.002.043); Alexander, Jones and Sinclair have presented narrow-band photometry of faint red stars (34.002.066).

The "Catalogue bibliographique de vitesses radiales stellaires", a continuation of the Abt-Biggs catalogue, is done by Barbier and Petit and is available at the CDS $(32.002 .035)$.

The Bright Star Catalogue has seen its radial velocities completed thanks to 106 southern stars observed by Beavers and Eitter (30.113.002), 139 B-type stars and 53 1ate-type stars measured by Andersen and Nordstroem (33.111.009, 33.111.010), and 790 late-type HR stars determined in the southern hemisphere by Andersen et al. (37.111.016). In a study of late-type dwarfs, radial velocities have been obtained by Dawson for 20 of the stars in Sanduleak's catalogue. The velocity dispersion and the space density appear to be "normal", i.e. there is no evidence for an excess density of low-velocity M dwarfs (30.111.007).

Radial velocities of 617 stars belonging to four stellar fields and $1127 \mathrm{ga}-$ lactic stars in the direction of the LMC have been measured with an objective prism technique by Fehrenbach and collaborators (32.111.001, 31.002.063). Radial velocities for 1246 stars measured at Herstmonceux and Kottania have been published by Woolley et a1. (31.002.054). Radial velocities have also been determined by Stetson for 124 southern early-type stars possibly having large space motions (34.111.005). of interest is also a compilation of radial velocities of 524 galactic planetary nebulae (34.002.034), and a list of radial velocities for 116 southern red stars published by Jones and Fisher (37.111.012).

Absolute proper motions of blue objects near the galactic pole have been derived by Schilbach with reference to 45 galaxies (32.111.015). Luyten and collaborators have produced numerous 1 ists of proper motions in the last three years: with Morris and Hill, a list of 7120 stars in the Hyades region (32.111.019), and a catalogue of 929 possible candidates for Hyades membership (31.111.033); with Hughes a sample of 374 faint stars near the north galactic pole (32.111.021), 280 faint stars (34.111.023), and a first supplement of 398 stars to the NLTT catalogue (31. $111.030)$.

Proper motions have been determined in the Orion association by Qian and Xu (30.111.018, 30.111.019), for 117 -type stars as well as for 211 stars in the north polar cap by Karimova and Pavlovskaya $(30.002 .050,33.111 .015)$, for AGK3R stars by Kalinina (33.002.023), in the field of the open cluster NGC 6913 by Gerts (31.111.010), in the area of the galactic cluster Coma Ber by Cova (32.111.012), and in the Hyades region by Stelmakh (33.111.016). A catalogue of proper motions from the Moscow PZT program $(33.111 .014)$ by Tochilina and a catalogue of relative proper motions and photographic UBV magnitudes in the region of $M 39$ by Platajs (34.002.037) have also been published. Wan, Mao and Ji have collected proper motions of RR Lyrae variables (30.002.081), and Fu and Gong have determined proper motions in R.A of 904 bright stars $(30.002 .080)$. See also proper motions studies 34.111 .008 and 33.111 .014 .

Spectroscopic, photometric and kinematic data for 488 white dwarfs were collected by Agayev et al. (31.002.012). A compilation prepared by Mennessier and co1- 
laborators of the most reliable kinematical data for stars of the HD and HDE, including positions and spectroscopic, photometric data is now available from the cDS $(31.002 .025)$.

The space distribution of stars in the region of Centaurus-crux has been investigated by McCuskey (31.002.025).

The galactic poles and centre-anticentre direction have been and continue to be directions of great interest for galactic studies. We should also notice surveys in course of progress to study stellar kinematics in the main meridional section of the Galaxy (see for example 34.155.018 and 33.155.060).

The relative parallaxes determined by five major general catalogue observatories have been intercompared to investigate their systematic differences by Lutz and collaborators (30.111.011). New trigonometric parallaxes appear in a few studies: by Breakiron et a1. (31.111.005), from the US naval faint stars programme (31.111.006), by Russel et a1. (31.111.007), by Kiselev (32.111.018), by Borgman and Lippincott (33.111.004), by Upgren and Kuzma (33.111.005), by Lippincott and Hershey (33.111.012), by van Altena and Sawada (34.111.011), and by Weis et al. $(34.111 .012)$.

We should further mention some important sources of basic information: The systematic reclassification of the $\mathrm{HD}$ stars on the MK system is progressing. Houk published the third volume of the Michigan catalogue of two-dimensional spectral types (declinations $-40^{\circ}$ to $\left.-26^{\circ}\right)(31.002 .126)$. Spectral types and luminosity classes have also been determined by Bartaya for 10396 stars in the Kapteyn Areas NN $2-43$ (34.002.018). The fourth revised edition of the Bright Star catalogue has been published by Hoffleit and Jaschek (31.002.077), as well as the fifth general catalogue of MK spectral classification (31.002.078), and a first supplement of the catalogue of star clusters and associations by Ruprecht et al. (31.002.111).

A new catalogue of stars suspected of variability containing data on 14810 variable stars which have not received definite nomenclature until 1980 has been published by Kukarkin and collaborators $(32.002 .039,32.002 .090)$.

of considerable interest for galactic structure studies are the numerous $\mathrm{CO}$ or $\mathrm{OH}$ data: A catalogue of CO radial velocities towards galactic HII regions by Blitz, Fich and Stark (32.002.004), CO observations of southern HII regions by de Graauw et a1. $(30.132 .035)$, Co observations around $1=45^{\circ}$ by Israel $(31.131 .059)$, Co observations of southern galactic-plane HII regions by White and Phillips ( 31.132 . 046), a CO survey covering $294^{\circ} \leq 1 \leq 354^{\circ}$ by McCutcheon, Robinson and Whiteoak (32.131.032), CO observations towards southern HII regions (33.132.040), towards $I=50^{\circ}$ and $72^{\circ}$ by Solomon, Stark and Sanders $(33.155 .031)$.

Recently acquired general information on $\mathrm{OH}, \mathrm{CO}$, $\mathrm{HI}$ and gamma ray surveys is available in "Surveys of the southern Galaxy" (34.012.023) or in "Regions of recent star formation" (31.012.002). In particular: a CO survey of 372 optical HII regions by Blitz et aI. (31.132.043), and HI and CO observations of distant HII regions in the galactic anticentre by Grayzeck et al. (31.132.044). See also 34.155.024, 34. $155.025,34.155 .038,34.155 .039,34.155 .043,34.155 .044,34.155 .045$.

From a southern survey of $\mathrm{OH}$ masers at $1612 \mathrm{MHz}$, the velocity and spatial distributions of the emission sources have been discussed with special reference to their kinematic properties and population type by Caswell et al. (30.131.107).

Let us also note numerous works of interest dealing with the distribution of molecular clouds and HII regions in the Galaxy and their kinematics (33.131.123, 
$33.131 .124,33.131 .126,33.132 .036,33.132 .037,33.132 .038)$ presented in "Kinematics, dynamics and structure of the Milky Way" (33.012.016).

High velocity HI streams, high velocity clouds or gas infalling on the Galaxy are detected and discussed in various studies: by Cohen $(30.131 .006,32.131 .021)$, Giovanneli (30.132.042), Mirabe1 (31.132.022, 32.131.150), Morras and Bajaja (32. $131.151,32.131 .264,33.131 .017)$, de Boer (33.131.138), and Lo and Clausen (34.155. 059).

The following HI surveys have been published: by 01 ano, Pöppel and Vieira $\left(320^{\circ} \leq 1 \leq 345^{\circ},+18^{\circ} \leq \mathrm{b} \leq+26^{\circ}\right.$ and $\left.346^{\circ} \leq 1 \leq 350^{\circ},+18^{\circ} \leq \mathrm{b} \leq+20^{\circ}\right)$, by Burton and Liszt $\left(349^{\circ} \leq 1 \leq 13^{\circ},-10^{\circ} \leq \mathrm{b} \leq 10^{\circ}\right)$, for $21 \mathrm{~cm}$ absorption at low latitudes towards 88 compact sources by Dickey et a1. (34.155.066), and in the halo of the Galaxy (34.155.057) by McGee, Newton and Morton. The $21 \mathrm{~cm}$ line radiation of neutral hydrogen in the central region of the Galaxy has been measured by Braunsfurth and Rohlfs (29.156.016), the region covered is $-1.5 \leq 1 \leq 195,-1.5 \leq \mathrm{b} \leq 195$. These authors have also carried out (in press) a survey of the innermost $21^{\circ}$ in longitude. Rohlfs, Kreitschmann and Siegman are preparing the publication of a $21 \mathrm{~cm}$ survey in the ranges $315^{\circ} \leq 1 \leq 340^{\circ},-5^{\circ} \leq \mathrm{b} \leq 5^{\circ}$.

We should further mention a catalogue of galactic supernovae remnants (34.002. 035), a catalogue of 0-type stars $(34.002 .101)$, the Lund catalogue of open-cluster parameters $(34.002 .127)$, the Geneva photometric-boxes catalogue on magnetic tape (31.002.064), a catalogue of $[\mathrm{Fe} / \mathrm{H}]$ determinations $(30.002 .001)$, and a catalogue of $\mathrm{H} \alpha$ observations $(30.002 .002)$.

Sakhibov and Smirnov (31.125.023) presented a complete survey of distance estimations for 57 galactic SNR's.

Weis has started a programme to determine photometric parallaxes of all stars of colour class $m$ from the NLTT Catalogue which are brighter than $V=14$ and north of the Celestial Equator. Results in VRI colours for 413 of these stars have already been published by Weis $(37.111 .014)$.

\section{B. INTRINSIC COLOURS AND INTERSTELLAR REDDENING}

Infrared properties of Mira-type variable and other cool stars as determined from JHKL photometry have been used by Feast and collaborators to establish periodcolour relations and to determine interstellar reddening $(32.122 .072)$.

Ultra-violet intrinsic colours of early-type stars have been presented from satellite observations and compared with a grid of stellar model atmospheres ( 30 . 113.003). For UV intrinsic colours, see also Carnochan (32.113.044), Wesselius (32. 113.059), Galecki et a1. (33.113.050), and Zorec et al. (34.113.011).

Cramer and Maeder, applying Geneva photometry, have classified $3600 \mathrm{~B}$ and Ap stars (30.113.032), and Cramer has determined intrinsic colours of B stars in the Geneva photometry (32.113.014). A comparison between the observed intrinsic colours $(b-y)_{0}$ and the calculated theoretical index $(b-y)$ has been carried out by Burkhart et al. for Ap stars (30.113.039). Wide-band photometric systems in the red and near infrared have been discussed by Straizys and collaborators in relation with the influence of reddening $(30.113 .056)$. Mean intrinsic $U-B$ and $B-V$ indices for population II stars as a function of temperature have been studied by Malyuto and Traat. Based on the method of "Geneva photometric boxes", Nicolet has determined a reddening of $E_{B-V}=0.04$ in the directions of both galacic poles (31.113.008). However, from uvby $\beta$ photometry of $5720-F$ stars in the south pole direction, Fadzean et al. have estimated a zero value for the interstellar extinction (34.113.023). 
Becker and Wooden have studied the space density of stars and interstellar extinction near $h$ and $x$ Persei, using RGU photometry (30.115.025).

$\mathrm{E}(\mathrm{b}-\mathrm{y})$ variations on scales smaller than $30^{\prime}$ have been investigated by Knude, using uvby $\beta$ observations in intermediate and low-latitude areas SA 128 and 156 (31. 113.090). With infra-red photometry, Castor and Simon constructed reddening-independent normal colours for O stars (33.113.010).

Calibrations of photometric indices of red giants in the old disk population for the determination of reddening, luminosity and metal abundance were discussed by Eggen (33.113.052) and are applied to determine the reddening, metal abundance and Iuminosity of high-luminosity G-type stars (34.113.002).

Intrinsic colours and absolute calibration values have been established between one and five microns by Koornneef $(34.113 .025)$.

U1traviolet interstellar extinction towards 1367 stars has been measured by Meyer and Savage in application of the ANS narrow-band photometry. Many stars have been isolated with significant deviations from the mean galactic extinction curve (30.131.023). Deviations from the normal absorption law are also presented from UBVRI photometry measurements in some dusty HII regions $(30,132.029)$.

A high-resolution optical survey of interstellar absorption lines, including line-of-sight measurements towards $3 \mathrm{C} 273$, the nucleus of $M 87$ and the cores of globular clusters allowed an estimation by Songaila, Cowie and York of the galactic halo column density $(30,131.027)$. The distribution of gas in the halo has also been studied by Blades et al., using the nuclei of two Seyfert galaxies as background probes (32.131.043,32.131.186), by de Boer and Savage, using a blue star in $M$ (33.131.022), and by using distant early-type stars $(32.131 .082,33.131 .168)$.

For UV absorption by interstellar gas at large distances from the galactic plane, see Savage and de Boer (30.131.043), and Pettini and West (32.131.082). The distribution of the interstellar gas in the galactic halo has been studied by Hobbs et al., using $21 \mathrm{~cm}$ emission lines and Lya absorption lines towards high-latitude $O B$ stars (32.155.060). From a high-latitude HI cloud with optical emission, Goerigk et al. found the ratio of HI column density to extinction to be about a factor of jo smaller than the standard value found from UV observations (33.131.101).

Deviations of galactic reddening from the cosecant relation have been discussed by Noonan $(30.131 .037)$.

Maps of reddening in the Galaxy, derived from $\mathrm{HI}$ and galaxy counts, have been established by Burstein and Heiles; these cover almost the whole galactic sphere with $\mathrm{b} 210^{\circ}$ (32.131.005). Based on uvby $\beta$ photometry of about 3450 bright A-F stars, a map of interstellar reddening within 300 pc was done by Perry and Johnston (32. 131.296). For interstellar reddening within $100 \mathrm{pc}$, see 32.131.309.

Interstellar extinction within $5 \mathrm{kpc}$ from the Sun in the direction of the galactic centre has been investigated by Ichikawa et al. (32.131.037). Interstellar light absorption in the Galaxy has also been investigated from the galactic latitude distribution of quasars by Nazarov and Khodyachikh (33.155.065), and of extragalactic objects by de Vaucouleurs and Buta (34.155.002). Hilditch, Hill and Barnes have established the interstellar reddening for the North Galactic pole region and uvby $\beta$ intrinsic colour calibration from a sample of 1000 stars with $b>75^{\circ}$ ( 34 . 131.023).

Golay et al. (1984) have used $1236^{\circ}$ fields near the galactic plane, photographed by balloon in the medium ultraviolet $(\lambda \cong 2000 \AA)$, to determine the distribution of interstellar extinction. 
Frisch and York have composed a synthesis map of ultraviolet observations of neutral interstellar gas from satellite observations of 140 stars, 10-3000 pc dis$\operatorname{tant}(34.131 .091)$.

A catalogue gives equivalent widths of the interstellar $2200 \AA$ band obtained from TD-1 spectrophotometric data $(34.131 .012)$.

Finally, we can mention reddening determinations in specific directions (34. 113.035; Orion 30.131.028; Taurus dark clouds 32.131.169; Cep OB 3 32.131.174; Cas $O$ OB 6 32.131.175; Cygnus 32.131.306; Aur OB-2, SCO OB-1 32.131.318; Carina neb. 34.131 .028 ).

\section{ABSOLUTE MAGNITUDES}

Using Strömgren photometry for Orion B stars, Anthony-Twarog has rediscussed the $\mathrm{HB}$ distance scale for B stars (32.111.004).

Systematic effects in trigonometric parallaxes have been searched for by Lutz et a1. through inter-observatory comparisons (30.111.011) or through comparisons with spectroscopic and cluster parallaxes (33.111.001).

Absolute magnitudes analysis have been carried out for Ap stars (30.115.001) by Grenier et al., Carbon stars $(30.115 .014)$ by Cohen et al., M supergiants (30. $115.015)$ by Elias et al., $\delta$ Scuti and $\delta$ Del-type stars $(30.115 .017)$ by Grenier et a1., OB stars $(30.115 .024)$ by Vega and Muzzio, G5-M3 stars near the giant branch (31.115.006) by Egret, Keenan and Heck, non-supergiant Be stars (31.115.012) by Kozok, $O$ and $B$ stars $(31.115 .035)$ by Divan and Zorec, F, G. K and M-type stars (32.115.020) by Mikami and Heck, M giants in the nuclear bulge of the Galaxy ( 32 . $155.003)$ by Frogel and Whitford, for nearby stars $(30.113 .017,34.155 .136)$ by Grenon and Rufener, B and Ap stars $(30.113 .032)$ by Cramer and Maeder, B-type supergiants (33.113.053) by zhang, 03 to 05 stars $(32.115 .022)$ by Underhil1.

Grenier et al. (1984) have extented their absolute magnitude calibration to norma1 stars.

Bolometric corrections have been determined by Habets and Heintze for the main-sequence stars (32.115.019).

The period-luminostiy relation for cepheids has been rediscussed in relation with infrared photometry by Madore et al. (30.122.040), McGonegal et al. (30.122. $111,31.122 .089,33.122 .147)$, and by welch et a1. $(34.122 .121)$; in relation with its zero point by Karimova and Pavlovskaya (30.122.081), and by Caldwel1 (34.122. 061); in relation with the surface brightness - colour relation by Ivanov (30.122. 061). For the cepheid PL relation, see also 32.122.128, 32.122.129, 34.122.032, and 34.122 .220 .

Stift has argued that the canonical cepheid PLC relation leads to an incorrect cosmic distance scale (32.122.017). In his classification of intrinsic variables, Eggen has discussed the cepheid domain (33.122.018). For a study of cepheid properties in the photometric Walraven system, see Coppola et al. (32.122.060).

Clube and Dawe followed some considerations arising from the faint absolute magnitude of halo RR Lyrae variables and proposed a modification of the PLC relation $(33.122 .139)$.

The surface brightness relation has been applied by Manduca et al. for the determination of absolute magnitudes of RR Lyrae stars $(30.122 .100)$. Luminosities of 
RR Lyrae variables in Osterhoff-group clusters have been studied by Cox et al. ( 31 . 122.073) (see also 30.154.011). Glass and Feast derived distances for 70 Mira variables in the Galactic Centre Windows by using the period-luminosity relation for Mira variables $(31.122 .001)$.

It was shown by Cousins that the surface-brightness parameter $F_{\mathrm{V}}$ can be obtained from VRI $I_{c}(30.113 .053)$. A new calibration of the Vilnius photometric system in spectral types and absolute magnitudes has been proposed by Straizys and collaborators $(30.113 .054,30.113 .055,32.113 .064)$, as well as for the Vilnius-Geneva system $(32.113 .066)$.

\section{THE STELLAR LUMINOSITY FUNCTION}

The degree of completeness of nearby stars and the stellar luminosity function have been analyzed by Upgren and Armandroff (30.111.017). Probst and $0^{\prime}$ Connell, from an infrared search for very low mass stars in binary systems with white dwarf primaries, found that the IMF declines steeply for $\mathrm{M}_{\mathrm{V}}>14$, and it appears unlikely that very low mass stars make a substantial contribution to the mass of either the disk or the massive halo components of the Galaxy $(31.126 .005,34.126 .015,34.126$. 020). Areas around 21 nearby stars have been scanned by Jameson et a1. (34.126.008) but no black dwarfs were found. The luminosity function in the solar neighbourhood was analyzed by Reid and Gilmore $(32.115 .008,32.115 .009)$. Form a purely photometric derivation of the stellar luminosity function to $\mathrm{M}_{\mathrm{v}}=+19$ (magnitude comparable to the minimum mass for thermonuclear burning), they conclude that the missing mass in the solar neighbourhood, if any, does not reside in main-sequence stars brighter than $M_{v} \approx+17$ mag.

From a complete sample of about 12500 stars brighter than $I=18$ towards the south galactic pole, Gilmore and Reid (33.155.015) have derived stellar luminosity function and density laws with distance from the galactic plane. They identified the 300 pc-scale-height old-disk component and a 1350 pc-scale-height "thick disk" component.

Kipp derived the stellar luminosity function in the direction of the south galactic pole by a statistical method using proper motions (32.031.551). Bisiacchi et al. have studied the mass function for massive stars (33.115.003). From UBV photometry in the anticentre direction, Morales Duran showed that the slope of the luminosity function at about $1 \mathrm{kpc}$ is the same as for the solar neighbourhood ( 31. 113.012). The space density and the luminosity function for the halo and disk populations in the test field SA 54 of the Basel halo program have been derived by Fenkart and Esin-Yilmaz. See also density and luminosity function in Cassiopeia by Fenkart and Kandemir (34.113.035), and a field in Centaurus by Spaenhauer and Fang $(31.155 .008)$.

From a proper motion study, a new determination of the halo luminosity density of the Galaxy was established by Richstone and Graham (30.155.007). From a deep blink survey of faint red objects towards the south galactic pole, Staller et al. obtain a surface density for $M$ dwarfs per square degree that is approximately twice as large as would be expected from Luyten's (1968) luminosity function (adopting a scale height of $260 \mathrm{pc})(31,155.003)$. The density distribution of faint stars in the direction of the north galactic pole has been analyzed from star counts in three-colour-photometry by Yoshii (32.155.034). The initial mass function (IMF) for stars more massive than $20 \mathrm{M}_{\odot}$ was derived by Garmany, Conti and Chiosi from a set of 750 o stars. Substantial variation in the IMF of the most massive stars has been found with varying distance to the galactic centre $(32.155 .061)$.

In an analysis of the observed colour distribution of faint stars, Bahcall, Schmidt and Soneira place strong upper limits on a possible intermediate population with $5 \leq M_{v} \leq 8$ in the galactic spheroid (33.155.012). 
Mezzeti et al. have estimated the influence of binarity on the local stellar mass density determination $(33.155 .102)$.

The IAU Colloquium No. 76 "The nearby stars and the stellar luminosity function" (34.012.065) offers an excellent review of the subject. In particular, we should mention the studies on the luminosity function of white dwarfs by Dahn et a1. (34.126.040), by Liebert et al. (34.126.041), by Ishida $(34.126 .042)$, and by Shipman (34.155.133); further, the search for brown dwarfs by Probst (34.126.043), a list of one thousand new $K$ and $M$ dwarfs brighter than the 13th magnitude by Stephenson (34.155.103), and the new limits on the surface density of $M$ dwarfs by Booshaar and Tyson (34.155.106). From the Two Micron Sky Survey, the space density distribution of late-type giants and supergiants in the solar vicinity (34.155.107) was derived by Ishida. In the context of an extensive survey of trigonometric parallaxes and proper motions in the region of the south galactic cap, Murray discussed the populations of red stars and the space density and kinematics of $K$ dwarfs (34.155.108). Stellar populations at higher galactic latitudes were discussed by Burer and Kaeser (34.155.111) and by Upgren and Philip (34.155.135). Schmidt studied the stellar luminosity function based on selected proper motion stars (34. 155.112), Wielen et al. the luminosity function of nearby stars (34.155.133), and Pritchet the luminosity function from star counts (34.155.114). Robertson discussed the luminosity function and kinematics of late $M$ dwarfs from an objective-prism survey (34.155.131). Smethells, from a sample of 185 red dwarfs selected in an objective-prism survey, has reanalyzed the luminosity function of early M dwarfs (34. i55.134). Tables and plots of luminosity functions were prepared by Philip and Upgren (34.155.137). Gilmore reported on an extensive analysis of the stellar distribution in the galactic spheroid and the faint end of the local luminosity function currently under way, using automated photographic photometry. Results concerning the luminosity function of main sequence stars are given $(34.155 .118)$.

Stellar populations and luminosity functions are discussed by Reid (34.155. $115)$ and Crézé (34.155.116). A comparative study of the Bahcal1-Sonaira Galaxy model with various observations has been carried out (34.155.119); all of the data are derived by a two-component model. with an exponential disk and a de Vaucouleurs spheroid and do not seem to confirm the fat-disk model proposed by Gilmore and Reid.

The compilation of a "Third catalogue of nearby stars" is in progress. Trigonometric parallaxes exceeding 0.039 of about 1900 stars are known in 1984 (G1iese and Jahreiß). Luminosity relations, mainly for red dwarfs, were calibrated for the data of Smethe11's stars (G1iese, 29.111.009), for Eggen's photometry of 1arge proper motion stars (G1iese, 31.111.004), and for the late Kuiper types (Jahreiß and Gliese, 32.036.022).

\section{E. REFERENCES}

Braunsfurth, E., Rohlfs, K.: 1984, Astron.Astrophys.Supp1. (in press). Golay, M., Cramer, N., Huguenin, D., Nicolet, B., Blecha, A.: 1984, Astrophys. Space Sci. (submitted).

Grenier, S., Gomez, A.E., Jaschek, C., Jaschek, M., Heck, A.: 1984, Astron, Astrophys. (submitted). 